\title{
Sodium valproate combined with levetiracetam in pediatric epilepsy and its influence on NSE, IL-6, hs-CRP and electroencephalogram improvement
}

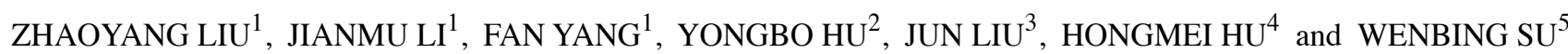 \\ Departments of ${ }^{1}$ Pediatrics, ${ }^{2}$ Gastrointestinal Surgery, ${ }^{3}$ Oncology, ${ }^{4}$ Ophthalmology and ${ }^{5}$ Gastrointestinal Medicine, \\ Xiantao First People's Hospital Affiliated to Yangtze University, Xiantao, Hubei 433000, P.R. China
}

Received November 20, 2019; Accepted December 10, 2019

DOI: $10.3892 /$ etm.2020.8916

\begin{abstract}
Efficacy of sodium valproate combined with levetiracetam (LEV) in pediatric epilepsy and its influence on neuron-specific enolase (NSE), interleukin-6 (IL-6) and high-sensitivity C-reactive protein (hs-CRP) as well as electroencephalogram (EEG) improvement were studied. Patients $(n=100)$ with pediatric epilepsy admitted to and treated in Xiantao First People's Hospital Affiliated to Yangtze University from December 2015 to 2018 were enrolled in this study and randomly divided into observation group $(n=50)$ and control group $(n=50)$. Sodium valproate was administered in the control group, and the treatment with LEV was combined with sodium valproate in the observation group. After 12 weeks the cognitive function of patients was assessed using the Mini-Mental State Examination (MMSE) scale, Montreal cognitive assessment (MoCA) scale and Wechsler Memory Scale-Revised in China (WMS-RC). The quality of life (QOL) of patients was evaluated with the QOL in epilepsy-31 inventory (QOLIE-31) scale and Barthel Index, and blood was drawn from the patients to detect the neurological function indicators [NSE and glial fibrillary acidic protein (GFAP)] and inflammatory indicators (IL-6, IL-2 and hs-CRP). After treatment, the incidence rates of adverse reactions notably declined in the observation group $(\mathrm{P}<0.05)$, and the improvement in the cognitive function in the observation group were both superior to those in the control group $(\mathrm{P}<0.05)$. Observation group had lowered content of NSE, GFAP, IL-6, hs-CRP and IL-2 ( $<<0.05)$, and $\alpha$ wave was markedly decreased, but $\theta$ and $\delta$ waves were notably increased in the observation group $(\mathrm{P}<0.05)$. In the treatment of pediatric epilepsy, sodium valproate combined with LEV produces
\end{abstract}

Correspondence to: Dr Wenbing Su, Department of Gastrointestinal Medicine, Xiantao First People's Hospital Affiliated to Yangtze University, 29 Middle Section of Mianyang Avenue, Xiantao, Hubei 433000, P.R. China

E-mail: zand2254@163.com

Key words: sodium valproate combined with levetiracetam, pediatric epilepsy, efficacy, NSE, IL-6, hs-CRP, electroencephalogram improvement better efficacy, fewer adverse reactions, significantly improves patients' QOL and notably lowers the content of NSE, IL-6 and hs-CRP with notable EEG improvement, so it is a safe and reliable treatment that is worth popularization.

\section{Introduction}

Epilepsy is quite a common nervous system disease even in adults. However, epilepsy has different incidence in different age groups, and its seizure frequency in children is obviously higher than that in adults (1). Epilepsy may be attributed to multiple factors, mainly including genetic abnormality, interruption of normal development and perinatal injury (2). According to the studies on the mental symptoms of children and young people experiencing epileptic seizures, epilepsy patients have a very high risk of mental disorder (3), including depression and anxiety (4). It was reported that the use of barbiturates can contribute to depression in childhood, but little is known about the overall incidence rate of depression and anxiety in epilepsy children and the determinants, which have not yet been deeply researched (5). Rutter et al (6) reported that mental disorder occurs in as many as $33 \%$ of epilepsy children, which was, however, not elaborated. Hoare (7) found that the incidence rate of behavior difficulty in epilepsy children is higher than that in diabetes children, but the incidence rates of depression and anxiety remain to be identified. Therefore, the cognitive disorders in epileptic child patients need to be further studied.

Valproate (VPA) and its derivants are widely applied in antiepileptic drugs (AEDs). As recommended by the International League Against Epilepsy, VPA is a potential candidate initial monotherapy for childhood absence epilepsy (8). The concerns regarding the use of VPA in reproductive women enable the shift of the prescription to novel AEDs, especially levetiracetam (LEV), lamotrigine and topiramate. LEV, a new generation of AED, has widely recognized efficacy and tolerance in treating epilepsy. Synaptic vesicle protein $2 \mathrm{~A}$ is an intracellular protein that serves as the binding site of LEA in the brain $(9,10)$. Prenatal exposure to lamotrigine has been confirmed to be able to significantly heighten the risk of neuropsychological function impairment compared to that in VPA in children, whether they are aged 4 or 5 years or of school age. However, there is little evidence for the risk caused 
by the exposure to LEV or topiramate. The infants exposed to LEV have consistent neurodevelopment with controls, and better development at the age of 1 and 3 years, compared with that of infants exposed to VPA $(11,12)$, suggesting that LEV has fewer adverse effects on epileptic children. In recent years, the importance of intellectual function in the judgment of agnosia and prognosis in epileptic patients has been better understood, and there have been increasingly more relevant research. Long-term use of AEDs is one of the important factors influencing cognitive function (13).

Aiming at the clinical problems, the electroencephalograms (EEGs) of the potential epilepsy patients are evaluated for several different purposes. Firstly, for the patients who recently had paroxysmal episodes, EEG evaluation may help identify whether these episodes are derived from epilepsy. Then for those newly diagnosed with epilepsy or suspected of having epilepsy, it is likely to raise the certainty of diagnosis, demonstrate the risk of epilepsy recurrence and conduce to confirming the presence of epilepsy syndrome. Moreover, EEG is used to verify the nature of epilepsy in the existing seizures, and more importantly, probably to confirm the precise presence of epilepsy syndrome in patients with drug-refractory epilepsy (14). Lastly, in drug-refractory focal epilepsy, EEG remains the gold standard for the preoperative diagnosis, which can be used to localize irritative zone and epileptic seizure zone, as the symbol of the extension of epileptogenic zone. In such a case, postoperative epileptic seizure freedom can be predicted using EEG as well. EEG has high time resolution and provides a very good overview, thus potently supporting the diagnosis of pediatric epilepsy (15).

The present study explored the efficacy of sodium valproate combined with LVE in pediatric epilepsy and its influences on neuron-specific enolase (NSE), interleukin-6 (IL-6) and high-sensitivity C-reactive protein (hs-CRP) as well as EEG improvement. The advantages of this regimen were comprehensively evaluated by observing the efficacy in patients, quality of life (QOL) score, serum indicators such as NSE, IL-6 and hs-CRP, and EEG improvement, so as to provide a theoretical foundation and experimental basis for the treatment of pediatric epilepsy patients.

\section{Patients and methods}

General information. A total of 100 patients with pediatric epilepsy, who were admitted to and treated in Xiantao First People's Hospital Affiliated to Yangtze University (Xiantao, China) from December 2015 to 2018, were enrolled as the subjects in this study and divided into observation group $(n=50)$ and control group $(n=50)$ by the randomized controlled method. Inclusion criteria: Patients diagnosed based on the diagnosis criteria that conform to the epilepsy syndrome classification in 2014 National Standardized Diagnosis,Treatment and Scientific Research of Epilepsy, those who voluntarily participated in the present study, those who signed the informed consent, those who received no treatment previously and those without allergy history of medications used in this study. Exclusion criteria: Patients who recently took drugs that affect growth and development as well as glucose and lipid metabolisms, those who used glucocorticoids for a long time, those with severe electrolyte disorder, or those with severe dysfunctions of the liver or kidney. All the clinical specimens in this experiment were collected with the approval of the Ethics Committee of the hospital and the patients as well as their family members, and the clinical trial regimen was approved by the Ethics Committee of Xiantao First People's Hospital Affiliated to Yangtze University. Table I shows the patients' clinical information collected at admission, including age, sex, weight, physical conditions and course of disease. There were no statistically significant differences in the general clinical information of patients between the two groups.

Treatment methods. Patients in the control group were given sodium valproate sustained-release tablets normally at the dose of $40 \mathrm{mg} / \mathrm{kg} / \mathrm{d}$, which was then gradually adjusted to $50 \mathrm{mg} / \mathrm{kg} / \mathrm{d}$, for 3 courses with 30 days as a course. In addition to the procedures in the control group, LEV treatment was performed in the observation group as follows: The initial dose was $20 \mathrm{mg} / \mathrm{kg} / \mathrm{d}$ and the dose was increased once every 5-7 days and maintained within $30 \mathrm{mg} / \mathrm{kg} / \mathrm{d}$. In case of epileptic seizure, the dose was increased by no more than $2 \mathrm{~g} /$ days, and the medications were taken twice for 3 consecutive months. During the whole medication, the administration was appropriately adjusted according to the disease changes in patients, and all the indicators were detected after the course of treatment.

Observation of efficacy in the two groups of patients. Efficacy was evaluated based on the following criteria: Control: After treatment, clinical symptoms and signs completely disappear without acute epileptic seizures in the epileptic children. Marked effectiveness: After treatment, the frequency of epileptic seizures declines by over $75 \%$, and the acute epileptic seizures can be controlled by drugs in epileptic children. Effectiveness: After treatment, the frequency of epileptic seizures is decreased by $50-70 \%$ in epileptic children. Ineffectiveness: After treatment, the frequency of epileptic seizures is decreased by $<50 \%$ in epileptic children. The number of patients at each grade was counted and recorded in detail.

Observation of adverse reactions in the two groups of patients. The adverse reactions were observed and recorded in the two groups of patients as follows: the adverse reactions, sleepiness, rash, gastrointestinal reaction and nausea, in the two groups of patients were recorded by specially-arranged medical staff, and the specific types of adverse reactions were counted and recorded in detail. Finally, the adverse reactions after treatment in the two groups of patients were summarized.

Cognitive function scoring. After treatment, the cognitive function of patients was assessed in the two groups using the Wechsler Memory Scale-Revised in China (WMS-RC). At 3 months after treatment, the Mini-Mental State Examination (MMSE) was performed by specialized neurologists, with the main examination items of orientation, memory, ability of mental arithmetic, short-term ability to listen and memorize and language and imitation abilities, and the higher the score is, the better the cognitive function will be (100 points in total). The cognitive function of the patients was assessed using the Montreal cognitive assessment (MoCA) scale with the total score of 30 points, and the higher scores correspond to better cognitive function. 
Table I. Clinical information of the patients.

\begin{tabular}{lcc}
\hline $\begin{array}{l}\text { Clinical } \\
\text { information }\end{array}$ & $\begin{array}{c}\text { Control } \\
\text { group }\end{array}$ & $\begin{array}{c}\text { Observation } \\
\text { group }\end{array}$ \\
\hline $\mathrm{n}$ & 50 & 50 \\
Male & 25 & 26 \\
Mean age (years) & $2 \pm 1.1$ & $2 \pm 1.3$ \\
Mean weight (kg) & $15.5 \pm 3.6$ & $16.1 \pm 3.2$ \\
BMI (kg/m ${ }^{2}$ ) & $20.9 \pm 1.0$ & $21.2 \pm 1.3$ \\
Course of disease (month) & 4 & 4.5 \\
\hline
\end{tabular}

QOL scoring. The QOL of the patients was scored using the QOL in epilepsy-31 inventory (QOLIE-31) scale which was properly revised according to Chinese cultural backgrounds and features. At the same time, their daily living abilities were evaluated using Barthel Index with the total score of 100 points, and the higher score denotes stronger daily living abilities and suggests higher QOL in patients. The scoring was conducted and elaborated in the two groups of patients by at least three medical staff members.

Detection of changes in the neurological function indicators NSE and glial fibrillary acidic protein (GFAP). Before and after course of treatment, $5 \mathrm{ml}$ of venous blood was drawn from the arm of the patients, respectively, placed in an Eppendorf (EP) tube containing anticoagulant and centrifuged at 2,000 $\mathrm{x} g$ and room temperature for $15 \mathrm{~min}$. The supernatant was collected to detect the changes in the content of serum neurological function indicators NSE and GFAP according to the instructions of the enzyme-linked immunosorbent assay (ELISA) kit (Nanjing Jiancheng Bioengineering Institute). The absorbance in each group was measured using a microplate reader.

Evaluation of changes in the content of serum $I L-2, I L-6$ and $h s-C R P$. After treatment, $5 \mathrm{ml}$ of venous blood was drawn from the arm of the patients, placed in an EP tube containing anticoagulant and centrifuged at 2,000 $\mathrm{x} g$ at room temperature for $15 \mathrm{~min}$. The supernatant was collected to detect serum inflammatory factors IL-2, IL-6 and hs-CRP in accordance with the operation instructions of the ELISA kit (Nanjing Jiancheng Bioengineering Institute). Finally, the absorbance in each group was measured using the microplate reader.

Detection of EEG improvement. After the third course of drug administration, EEG examination was performed. The electrodes were placed according the international 10-20 system, with the monitoring duration no shorter than $4 \mathrm{~h}$, including wake-up and sleep periods. With the number of spike waves per minute, namely spike wave index, as the statistical indicator, the mean frequency of $\alpha$ wave and the number of $\beta, \theta$ and $\delta$ waves was observed and recorded.

Statistical analysis. The raw experimental data recorded were processed using SPSS 20.0 analysis software, and subjected to multiple comparisons. $\chi^{2}$ test was employed for the percentage. The experimental results obtained were expressed as mean \pm standard deviation (mean $\pm \mathrm{SD}$ ), and $\mathrm{P}<0.05$ indicated

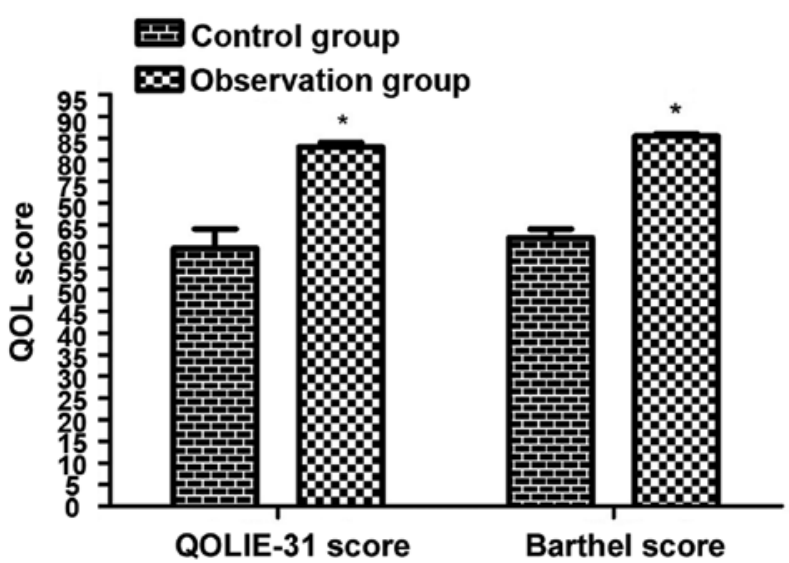

Figure 1. QOL score. Observation group exhibits a remarkably higher QOL score than control group $(\mathrm{P}<0.05) .{ }^{*} \mathrm{P}<0.05$ vs. control group. QOL, quality of life.

statistically significant differences. Histograms were plotted using GraphPad Prism 6.0.

\section{Results}

Clinical efficacy of patients. As shown in Table II, there was a statistically significant difference in the total effective rate of clinical treatment between the observation group and the control group (96 vs. 70\%) $(\mathrm{P}<0.05)$.

Adverse reactions in the two groups of patients. According to the statistical results (Table III), the cases of sleepiness, rash, gastrointestinal reaction and nausea declined markedly in the observation group $(\mathrm{P}<0.05)$.

Cognitive function of patients in both groups. Observation group had notably higher WMS-RC, MMSE and MoCA scores than the control group $(\mathrm{P}<0.05)$ (Table IV), suggesting that the cognitive function was significantly improved.

QOL score. As shown in Fig. 1, the QOL score in the observation group was remarkably higher than that in the control group $(\mathrm{P}<0.05)$.

Serum NSE and GFAP content. According to the detection results (Table V), there was no statistically significant difference in the content ratio of NSE/GFAP before treatment ( $p>0.05$ ), and after treatment, the content of NSE and GFAP was obviously lowered in both groups of patients, but the decline in the observation group was dramatically greater than that in the control group $(\mathrm{P}<0.05)$.

Serum inflammatory factors detected via ELISA. The content of IL-2, IL-6 and hs-CRP was notably reduced in observation group $(\mathrm{P}<0.05)$ (Fig. 2), implying that the treatment with sodium valproate combined with LEV can significantly inhibit the production of inflammatory factors.

EEG findings. As shown in Table VI, observation group exhibited substantially decreased $\alpha$ wave $(\mathrm{P}<0.05)$, but notably increased $\theta$ and $\delta$ waves $(\mathrm{P}<0.05)$. 
Table II. Efficacy.

\begin{tabular}{lccccc}
\hline Group & Control & Marked effectiveness & Effectiveness & Ineffectiveness & Total effective rate \\
\hline Control group & 11 & 14 & 10 & 15 & 70 \\
Observation group & $16^{\mathrm{a}}$ & $20^{\mathrm{a}}$ & $12^{\mathrm{a}}$ & $2^{\mathrm{a}}$ & $96^{\mathrm{a}}$ \\
\hline
\end{tabular}

There is a statistically significant difference in the total effective rate of clinical treatment between the observation group and the control group (96 vs. $70 \%)(\mathrm{P}<0.05)$. ${ }^{\mathrm{P}}<0.05$ vs. control group.

Table III. Adverse reactions in the two groups of patients.

\begin{tabular}{lccccc}
\hline Group & Sleepiness & Rash & Gastrointestinal reaction & Nausea & Adverse reaction rate (\%) \\
\hline Control group & 3 & 2 & 4 & 5 & 28 \\
Observation group & 1 & 1 & 2 & 1 & $10^{\mathrm{a}}$ \\
\hline
\end{tabular}

Observation group has substantially decreased cases of sleepiness, rash, gastrointestinal reaction and nausea $(\mathrm{P}<0.05)$. ${ }^{\text {a }}<0.05$ vs. control group.

Table IV. Cognitive function of patients in both groups.

\begin{tabular}{lccc}
\hline Group & $\begin{array}{c}\text { WMS-RC } \\
\text { score }^{\mathrm{a}}\end{array}$ & $\begin{array}{c}\text { MMSE } \\
\text { score }\end{array}$ & $\begin{array}{c}\text { MoCA } \\
\text { score }\end{array}$ \\
\hline Control group & $78.4 \pm 2.4$ & $71.7 \pm 2.3$ & $21.5 \pm 1.9$ \\
Observation group & $90.8 \pm 2.6^{\mathrm{a}}$ & $89.5 \pm 2.1^{\mathrm{a}}$ & $27.9 \pm 2.0^{\mathrm{a}}$ \\
\hline
\end{tabular}

The WMS-RC, MMSE and MoCA scores in observation group are markedly higher than control group $(\mathrm{P}<0.05)$. ${ }^{a} \mathrm{P}<0.05$ vs. control group. WMS-RC, Wechsler Memory Scale-Revised in China; MMSE, Mini-Mental State Examination; MoCA, Montreal cognitive assessment.

Table V. Serum and GFAP content.

\begin{tabular}{lcc}
\hline Group & NSE $(\mathrm{ng} / \mathrm{ml})$ & GFAP $(\mathrm{pg} / \mathrm{ml})$ \\
\hline Control group & & \\
Before treatment & $15.4 \pm 1.2$ & $88.1 \pm 2.7$ \\
After treatment & $8.2 \pm 2.1^{\mathrm{a}}$ & $30.7 \pm 2.1^{\mathrm{a}}$ \\
Control group & & \\
Before treatment & $14.9 \pm 1.7$ & $91.7 \pm 1.9$ \\
After treatment & $5.7 \pm 2.7^{\mathrm{a}, \mathrm{b}}$ & $19.4 \pm 1.3^{\mathrm{a}, \mathrm{b}}$ \\
\hline
\end{tabular}

After treatment, the content of NSE and GFAP was reduced in both groups of patients, but the decline in the observation group is much greater than that in the control group $(\mathrm{P}<0.05)$. ${ }^{\mathrm{a}} \mathrm{P}<0.05$ vs. before treatment; ${ }^{\mathrm{a}, \mathrm{b}} \mathrm{P}<0.05$ vs. control group at the same time-point. NSE, neuron-specific enolase; GFAP, glial fibrillary acidic protein.

\section{Discussion}

Epilepsy is a common neurological syndrome characterized by complex etiology and recurrent seizures in childhood, which also refers to the convulsive seizure caused by paroxysmal
Table VI. EEG findings.

\begin{tabular}{lcccc}
\hline Group & $\alpha$ & $\theta$ & $\beta$ & $\delta$ \\
\hline $\begin{array}{l}\text { Control group } \\
\text { Observation group }\end{array}$ & $12.4 \pm 2.4$ & $21.7 \pm 2.3$ & $11.5 \pm 1.9$ & $17.5 \pm 1.9$ \\
& & $29.5 \pm 2.1^{\mathrm{a}}$ & $10.9 \pm 2.0$ & $25.5 \pm 1.9^{\mathrm{a}}$ \\
\hline$\alpha$ wave declines substantially $(\mathrm{P}<0.05)$, but $\theta$ and $\delta$ & waves were \\
increased notably in the observation group $(\mathrm{P}<0.05)$. & ${ }^{a} \mathrm{P}<0.05$ vs. \\
control group. EEG, electroencephalogram.
\end{tabular}

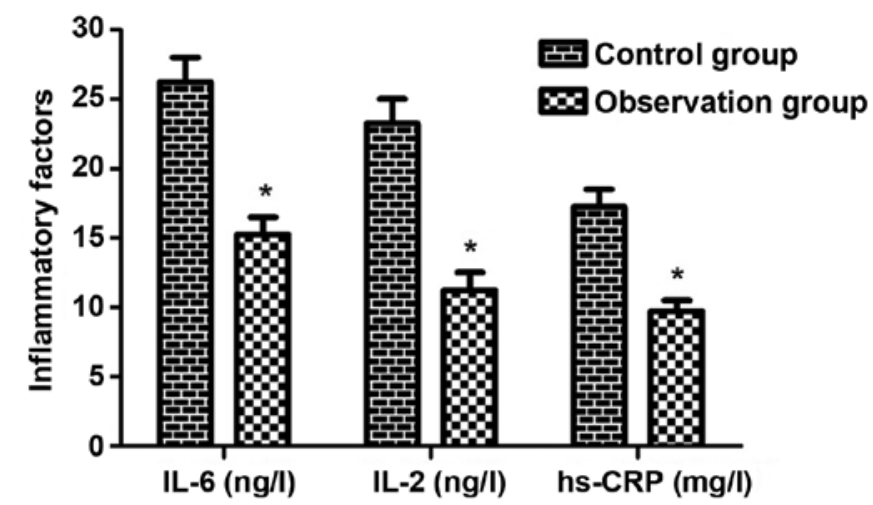

Figure 2. Serum IL-2, IL-6 and hs-CRP content. The content of IL-2, IL-6 and hs-CRP declined in observation group $(\mathrm{P}<0.05)$. ${ }^{*} \mathrm{P}<0.05$ vs. control group. IL, interleukin; hs-CRP, high-sensitivity C-reactive protein.

and temporary brain dysfunction. It can be classified into primary and secondary types according to etiology, with the clinical manifestations of recurrent jerk and transient abnormalities in consciousness, sense and emotion $(16,17)$. In the present study, the efficacy of sodium valproate combined with LEV in pediatric epilepsy and its influences on NSE, IL-6 and hs-CRP as well as EEG improvement were observed. Based on the results, there was a statistically significant difference in 
the total effective rate of clinical treatment between observation group and control group (96 vs. 70\%), and observation group had substantially reduced number of cases of sleepiness, rash, gastrointestinal reaction and nausea. A study found that the cognitive ability of epilepsy children of school age exposed to LEV, topiramate or sodium valproate can be improved dramatically (18), and it was found in the present study that the WMS-RC, MMSE and MoCA scores in the observation group were notably higher than those in the control group, suggesting that cognitive function was remarkably improved. Additionally, the QOL score in observation group was remarkably higher than that in the control group. The above findings in this study are similar to those in the previous study (19).

Currently, NSE is a commonly used indicator for evaluating cranial nerve function impairment in epileptic children, and it is a key enzyme with enolase activity in glucose metabolism, which is specifically present in neurons. When cerebral neurons are damaged, the activity of NSE will be remarkably enhanced, so the elevation of serum NSE can be taken as a highly specific and sensitive biochemical indicator reflecting neuronal damage (20). GFAP, a specific astrocyte marker, is expressed in the central nervous system. The high expression of GFAP can damage various aspects of the central nervous system (21), and the regulation of cognitive function, namely information reception, integration and transmission, is associated with the functions of the neurons and astrocytes in the cerebral cortex. According to the findings in the present study, before treatment, there was no statistically significant difference in the content ratio of NSE/GFAP, and after treatment, the content of NSE and GFAP was obviously reduced in both groups of patients, but the decline in the observation group was much greater than that in the control group. As an acute-phase protein, hs-CRP was confirmed to be increased in diabetes patients (22). However, whether its content is raised in patients with pediatric epilepsy remains to be further studied. The activation of inflammatory factors and generation of cytokines as well as increase in oxidative stress may cause tissue damage, and are related to the evolution of epilepsy, namely inflammation probably promotes the worsening of disease in epileptic patients (23). In this study, it was discovered that the content of IL-2, IL-6 and hs-CRP declined notably in observation group, implying that the treatment with sodium valproate combined with LEV can significantly inhibit the production of inflammatory factors. A study demonstrated that the increases in the absolute power and relative power (RP) of $\delta$ and $\theta$ waves are observed in EEGs of epilepsy children, while the RP of $\alpha$ wave is decreased. Besides, routine EEGs reveal interictal discharge such as spikes, multiple spikes and sharp waves (24), which is a rare activity in children with normal development (25). The results of this study showed that $\alpha$ wave declined substantially, but $\theta$ and $\delta$ waves were increased notably in the observation group, similarly to previous studies. The present study proved through a series of experiments that sodium valproate combined with LEV has favorable efficacy in pediatric epilepsy, and can substantially lower the levels of NSE, IL-6 and hs-CRP, and improve EEG indicators.

In conclusion, it was found through the various experiments in this study that sodium valproate combined with LEV can notably improve the pathology, cognitive function and QOL of patients, with a favorable overall effect. The present study provides a theoretical basis for the prevention and treatment of pediatric epilepsy and a novel idea for the forthcoming further research.

\section{Acknowledgements}

Not applicable.

\section{Funding}

No funding was received.

\section{Availability of data and materials}

The datasets used and/or analyzed during the current study are available from the corresponding author on reasonable request.

\section{Authors' contributions}

ZL wrote the manuscript. ZL and JiL collected and analyzed the general data of patients. FY and YH were responsible for the observation of efficacy. JuL and $\mathrm{HH}$ assisted with the cognitive function scoring and QOL scoring. ZL and WS detected EEG improvement. All authors read and approved the final manuscript.

\section{Ethics approval and consent to participate}

The study was approved by the Ethics Committee of Xiantao First People's Hospital Affiliated to Yangtze University (Xiantao, China) and informed consents were signed by the patients and/or guardians.

\section{Patient consent for publication}

Not applicable.

\section{Competing interests}

The authors declare that they have no competing interests.

\section{References}

1. Stafstrom CE, Moshé SL, Swann JW, Nehlig A, Jacobs MP and Schwartzkroin PA: Models of pediatric epilepsies: Strategies and opportunities. Epilepsia 47: 1407-1414, 2006.

2. Harvey AS, Cross JH, Shinnar S and Mathern GW; ILAE Pediatric Epilepsy Surgery Survey Taskforce: Defining the spectrum of international practice in pediatric epilepsy surgery patients. Epilepsia 49: 146-155, 2008.

3. Ettinger AB, Weisbrot DM, Nolan EE, Gadow KD, Vitale SA, Andriola MR, Lenn NJ, Novak GP and Hermann BP: Symptoms of depression and anxiety in pediatric epilepsy patients. Epilepsia 39: 595-599, 1998.

4. Becker AJ, Blümcke I, Urbach H, Hans V and Majores M: Molecular neuropathology of epilepsy-associated glioneuronal malformations. J Neuropathol Exp Neurol 65: 99-108, 2006.

5. Cross JH, Jayakar P, Nordli D, Delalande O, Duchowny M, Wieser HG, Guerrini R and Mathern GW; International League against Epilepsy, Subcommission for Paediatric Epilepsy Surgery; Commissions of Neurosurgery and Paediatrics: Proposed criteria for referral and evaluation of children for epilepsy surgery: Recommendations of the Subcommission for Pediatric Epilepsy Surgery. Epilepsia 47: 952-959, 2006.

6. Rutter M, Graham P and Yule WA: A neuropsychiatric study in childhood. Arch Dis Child 46: 577, 1971. 
7. Hoare P: The development of psychiatric disorder among schoolchildren with epilepsy. Dev Med Child Neurol 26: 3-13, 1984.

8. Ackers R, Besag FM, Wade A, Murray ML and Wong IC: Changing trends in antiepileptic drug prescribing in girls of child-bearing potential. Arch Dis Child 94: 443-447, 2009.

9. Vigevano F: Levetiracetam in pediatrics. J Child Neurol 20: 87-93, 2005.

10. De Smedt T, Raedt R, Vonck K and Boon P: Levetiracetam: Part II, the clinical profile of a novel anticonvulsant drug. CNS Drug Rev 13: 57-78, 2007.

11. Wen X, Meador KJ and Hartzema A: Antiepileptic drug use by pregnant women enrolled in Florida Medicaid. Neurology 84: 944-950, 2015.

12. Baker GA, Bromley RL, Briggs M, Cheyne CP, Cohen MJ, García-Fiñana M, Gummery A, Kneen R, Loring DW, Mawer G, et al; Liverpool and Manchester Neurodevelopment Group: IQ at 6 years after in utero exposure to antiepileptic drugs: A controlled cohort study. Neurology 84: 382-390, 2015.

13. Morrow J, Russell A, Guthrie E, Parsons L, Robertson I, Waddell R, Irwin B, McGivern RC, Morrison PJ and Craig J: Malformation risks of antiepileptic drugs in pregnancy: A prospective study from the UK Epilepsy and Pregnancy Register. J Neurol Neurosurg Psychiatry 77: 193-198, 2006.

14. Rosenow F and Lüders H: Presurgical evaluation of epilepsy. Brain 124: 1683-1700, 2001.

15. Tao JX, Ray A, Hawes-Ebersole S and Ebersole JS: Intracranial EEG substrates of scalp EEG interictal spikes. Epilepsia 46: 669-676, 2005.

16. Goldlust IS, Hermetz KE, Catalano LM, Barfield RT, Cozad R, Wynn G, Ozdemir AC, Conneely KN, Mulle JG, Dharamrup S, et al; Unique Rare Chromosome Disorder Support Group: Mouse model implicates GNB3 duplication in a childhood obesity syndrome. Proc Natl Acad Sci USA 110: 14990-14994, 2013.

17. Sakkalis V, Doru Giurc Neanu C, Xanthopoulos P, Zervakis ME, Tsiaras V, Yang Y, Karakonstantaki E and Micheloyannis S: Assessment of linear and nonlinear synchronization measures for analyzing EEG in a mild epileptic paradigm. IEEE Trans Inf Technol Biomed 13: 433-441, 2009.
18. Bromley RL, Calderbank R, Cheyne CP, Rooney C, Trayner P, Clayton-Smith J, García-Fiñana M, Irwin B, Morrow JI, Shallcross R, et al; UK Epilepsy and Pregnancy Register: Cognition in school-age children exposed to levetiracetam, topiramate, or sodium valproate. Neurology 87: 1943-1953, 2016.

19. Tumay Y, Altun Y, Ekmekci K and Ozkul Y: The effects of levetiracetam, carbamazepine, and sodium valproate on P100 and P300 in epileptic patients. Clin Neuropharmacol 36: 55-58, 2013.

20. Akcan A, Akyildiz H, Deneme MA, Akgun H and Aritas Y: Granulomatous lobular mastitis: A complex diagnostic and therapeutic problem. World J Surg 30: 1403-1409, 2006.

21. Takeda K, Sawamura S, Tamai H, Sekiyama H and Hanaoka K: Role for cyclooxygenase 2 in the development and maintenance of neuropathic pain and spinal glial activation. Anesthesiology 103: 837-844, 2005.

22. Karantza MV, Mittelman SD, Dorey F, Samie S, Kaiserman K, Halvorson M and Kaufman FR: Relationship of highly sensitive C-reactive protein and lipid levels in adolescents with type 1 diabetes mellitus. Pediatr Diabetes 9: 122-126, 2008.

23. Vezzani A: Inflammation and epilepsy. Epilepsy Curr 5: 1-6, 2005.

24. Cantor DS and Chabot R: QEEG studies in the assessment and treatment of childhood disorders. Clin EEG Neurosci 40: 113-121, 2009.

25. Porras-Kattz E, Harmony T, Ricardo-Garcell J, Galán L, Fernández T, Prado-Alcalá R, Avecilla-Ramírez G, Sánchez-Moreno L, Barrera-Reséndiz J, Corsi-Cabrera M, et al: Magnesium valproate in learning disabled children with interictal paroxysmal EEG patterns: Preliminary report. Neurosci Lett 492: 99-104, 2011.

This work is licensed under a Creative Commons Attribution-NonCommercial-NoDerivatives 4.0 International (CC BY-NC-ND 4.0) License. 